Check for updates

Cite this: Mater. Chem. Front., 2020, 4, 140

Received 13th July 2019

Accepted 20th September 2019

DOI: $10.1039 / c 9 q m 00458 \mathrm{k}$

rsc.li/frontiers-materials

\title{
Recent progress of electrowetting for droplet manipulation: from wetting to superwetting systems
}

\author{
Peicheng Teng, ${ }^{\mathrm{ab}}$ Dongliang Tian, ${ }^{* \mathrm{c}}$ Haiyan Fu*d and Shutao Wang $\mathbb{( D}^{\mathrm{ab}}$
}

\begin{abstract}
Many engineering and industrial technologies as well as biological processes are closely related to surface wettability. The control of surface wettability attracts much attention due to the prospective applications based on liquid and droplet manipulation. However, the dynamic modification of wetting is still a challenging issue both in theoretical models and practical applications. Electrowetting (EW) is one of the most widely used methods for controlling the wettability of liquids due to its fast responding speed, large switching range and excellent durability. Since Gabriel Lippmann first discovered the electrocapillary (ECP) phenomenon in 1875, which is a precursor of electrowetting, there has been considerable progress in this field, especially accompanied with the development of wettability and interface science. In this paper, we review the developments of EW for droplet manipulation in view of wetting to superwetting systems over the past decades, including theoretical basis, electrodes and fluid systems, droplet manipulation and special phenomena induced by EW. Finally, we discuss several difficult challenges in this field and provide future research directions from our perspective.
\end{abstract}

\section{Introduction}

Wetting is a common surface phenomenon in many engineering and technology fields, such as chemicals, materials, and biomedical areas. Over the past 200 years, studies on static wetting have developed worldwide, and innumerable great achievements have been made in this field. As a pioneer, Thomas Young defined surface wettability by proposing the concept of the contact angle (CA). Since then, engineers and scientists from different fields have systematically studied materials with various wettabilities and developed functional surfaces with special capabilities, such as superhydrophilic surfaces (water contact angle (WCA) less than $5^{\circ}$ ) and superhydrophobic surfaces (WCA $>150^{\circ}$ ). In addition, static wetting models for explaining the wetting behaviours on these surfaces have been well established. However, the dynamic regulation of the wetting properties on these surfaces

\footnotetext{
${ }^{a}$ Key Laboratory of Bio-Inspired Materials and Interfacial Science, CAS Center for Excellence in Nanoscience, Technical Institute of Physics and Chemistry, Chinese Academy of Sciences, Beijing, P. R. China

${ }^{b}$ University of Chinese Academy of Sciences, Beijing, P. R. China

${ }^{c}$ Key Laboratory of Bio-Inspired Smart Interfacial Science and Technology of Ministry of Education, Beijing Advanced Innovation Center for Biomedical Engineering, School of Chemistry, Beihang University, Beijing, P. R. China. E-mail: tiandl@buaa.edu.cn

${ }^{d}$ School of Information Science and Technology, Hainan Normal University, Haikou, Hainan, P. R. China. E-mail: fuhaiyan@hainnu.edu.cn
}

is still a challenging issue. Functional surfaces with controllable wettability, which can respond to external stimuli, have attracted enormous interest due to the prospective applications based on liquid and droplet manipulation. To date, various methods have been developed to manipulate droplets, including optical, ${ }^{1,2}$ magnetic, ${ }^{3,4}$ surface acoustic wave $e^{5,6}$ and thermal capillary ${ }^{7,8}$ techniques. Nevertheless, all the methods mentioned above have different defects, which limit their practical applications. For instance, optical methods are associated with relatively low throughput, and the lasers used in some systems may lead to biocompatibility problems. Magnetic actuation of droplets is a comparably convenient method to manipulate droplet movement. However, this approach requires the addition of magnetic nanoparticles to the droplet. Moving droplets with surface acoustic waves can cause a single droplet to orientate along the microchannel path in a non-invasive manner; however, this requires specially designed microchannels with satisfactory acoustic reflectivity. Also, a droplet floating on another immiscible liquid can be manipulated by thermal capillary waves with the help of a low gravity environment.

Compared to the above methods, electrowetting (EW) is one of the most widely used ways for controlling the wettability of liquids; this is mainly because of its fast response speed (several milliseconds), large switching range (several tens of degrees), excellent durability (hundreds of thousands of switching cycles) and low energy consumption (10-100 $\mu \mathrm{W})$. As a consequence, EW is very important in a host of practical applications, such as 

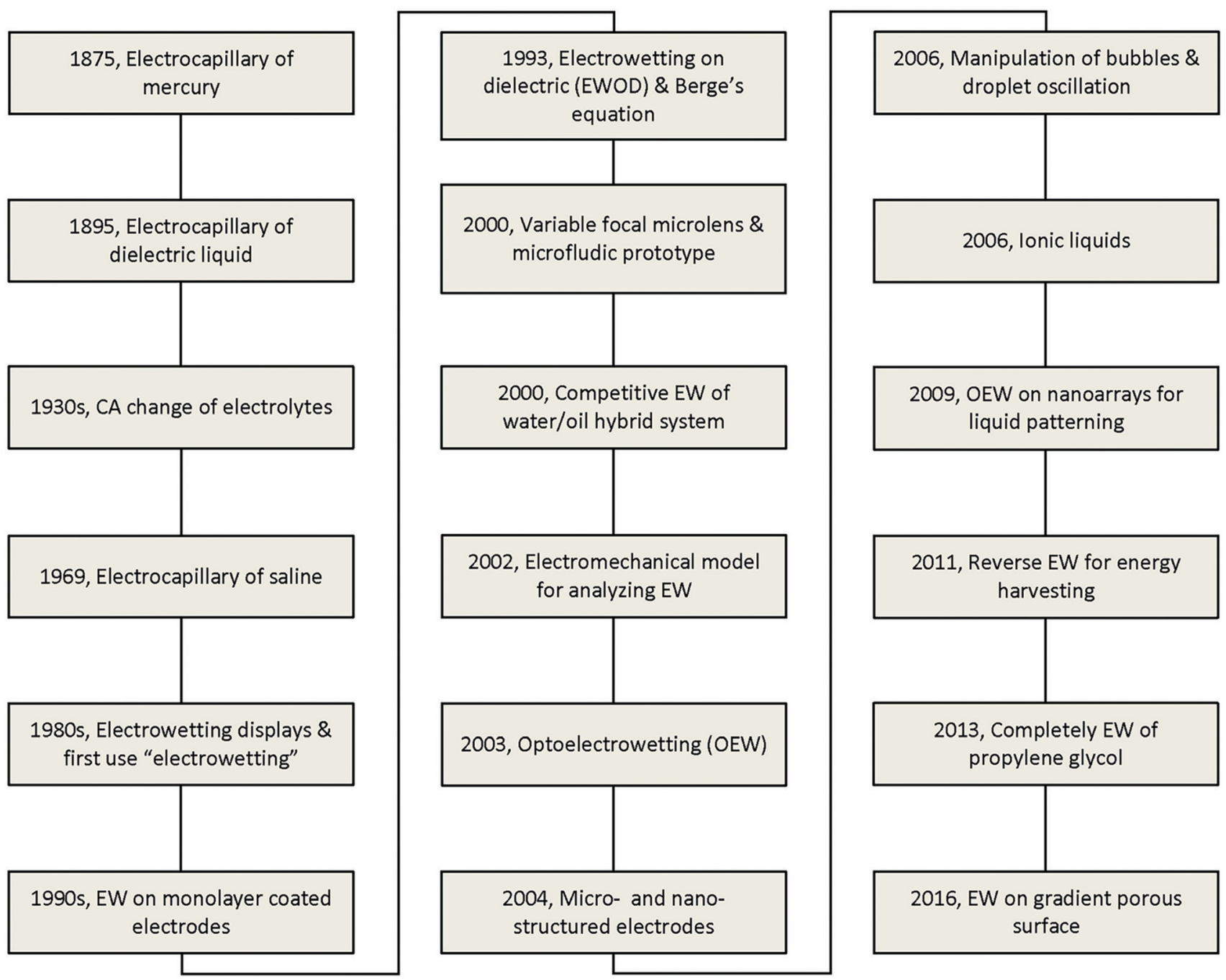

Fig. 1 Chart of the major discoveries and developments in EW.

microfluidics, variable-focus microlenses, and other microelectromechanical systems (MEMS).

The major developments in EW are summarized in Fig. 1. The earliest reports related to EW can be traced back to 1875 . In that year, G. Lippmann published his pioneering research on the electrocapillary effect, ${ }^{9}$ which is the earliest comprehensive investigation in this field. Briefly, by decreasing the surface charge between mercury and an electrolyte which was initially positive, it was found that the capillary depression of the mercury and hence the interfacial tension firstly increased to the maximum and then decreased. Based on this, Lippmann proposed the formula of electrocapillarity, that is, the famous Lippmann equation:

$$
\left(\frac{\partial \gamma}{\partial E}\right)_{\mu}=-\sigma
$$

where $\gamma$ is the liquid-gas surface tension, $E$ is the applied potential and $\sigma$ is the amount of charge per square centimetre at the interface of two phases. The relationship between the surface tension and electric field intensity is given in eqn (1).
Based on this, electrocapillarity enables the measurement of first-grade information at liquid interfaces. Another classic study that deserves to be mentioned is Pellat's experiment. ${ }^{10}$ He observed electrocapillarity on a dielectric liquid, now known as the dielectrophoresis (DEP) effect. Two vertical, parallel-plate electrodes were dipped into a pool of dielectric fluid. Upon applying an electric field, the liquid rose up between the two plates. Thereafter, research on EW focused on systems of aqueous electrolytes on bare metal. In the 1930s, A. Frumkin, the Russian pioneer of electrochemistry, reported CA changes of electrolytes on a polarized metal surface. ${ }^{11-13}$ Although these studies had a strong impact on electrochemistry at the time, insufficient attention was paid to EW. In the 1980s, G. Beni et al. reported several applications of the actuation of mercury droplets, including displays ${ }^{14,15}$ and optical switches. ${ }^{16,17}$ Additionally, they first introduced the terminology of "electrowetting" in their published paper, which is still used worldwide today. In the 1990s, J. Sondag-Huethorst and L. Fokkink ${ }^{18,19}$ investigated EW on alkylthiol-modified gold electrodes. The CA of a saline droplet decreased from $74^{\circ}$ to $\sim 50^{\circ}$ under an applied voltage of less than $1 \mathrm{~V}$. 
However, all the studies mentioned above remained far from practical application due to the involvement of toxic mercury or the inevitable electrolysis, beyond hundreds of millivolts. Then, in 1993, B. Berge et al. ${ }^{20}$ proposed the idea of covering dielectric films on plate electrodes to eliminate the problem of electrolysis. In brief, for a polymer film covering a steel disc, the CA decreased to about $30^{\circ}$ on PET and $70^{\circ}$ on PTFE under applied voltages of $200 \mathrm{~V}$ and $1000 \mathrm{~V}$, respectively. This effect is known as electrowetting on insulator coated electrodes (EICE) or electrowetting on a dielectric (EWOD), the latter of which is used more widely. Since then, almost all published papers about EW have utilized this process due to the large amplitude of the CA change, simplicity of fabrication, and low cost of the materials. This process directly contributed to the outbreak of research on EW in the early 2000s and was greatly important in facilitating the development of EW. Two main practical applications of EW emerged in 2000, namely variable focal lenses ${ }^{21}$ and microfluidic prototypes. ${ }^{22}$ Meanwhile, C. Oehr et al. ${ }^{23}$ systemically studied the EW characteristics of water/oil hybrid systems and proposed the competitive EW effect at the interface of two immiscible liquids. However, there was still no recognized mechanism until T. B. Jones ${ }^{24}$ reported that EW is essentially an electromechanical process. Subsequently, certain functional materials were introduced in EWOD to enhance its performance and enrich its applications. For example, C. Kim et al. ${ }^{25}$ first proposed optoelectrowetting for optical actuation of liquid droplets. Also, micro- and nano-structured electrodes were first introduced by T. N. Krupenkin et al. ${ }^{26}$ Other interesting behaviours reported later included bubble manipulation in an aqueous environment $^{27}$ and droplet oscillation under an AC electric field. ${ }^{28}$ Ionic liquids, a type of non-aqueous conducting liquid, have also been introduced in EWOD. ${ }^{29}$ Our group ${ }^{30}$ proposed photoelectric cooperative wetting (PCW) on nanoarrays for liquid patterning. Additionally, a novel energy harvesting system based on reverse EW was reported. ${ }^{31}$ Complete EW of propylene glycol, which involves a transition of EW from partial to complete wetting on a common planar electrode, was also reported in $2013 .{ }^{32}$ Recently, our group ${ }^{33}$ demonstrated that $\mathrm{EW}$ on a gradient porous surface can be used for liquid transport. With the cooperative effects of an electric field and gradient surface energy, this method can continuously transfer oil droplets underwater by directional motion.

With the development of surface wettability and materials science, many new research results on EW have been achieved. In this review, we aim to emphasize recent progress and breakthroughs in EW over the past decades. In Section 2, we give a brief introduction to the theoretical basis because the early theoretical developments were already comprehensively reviewed by F. Mugele and J. Baret. ${ }^{34}$ In Section 3, the development of fluid systems and electrodes is reviewed to demonstrate the accomplishments and contributions to practical applications. In Section 4, we summarize different modes of droplet manipulation, including transfer, oscillation, capillary bridge, permeation and bouncing. Other special phenomena induced by EW, such as a controllable Cheerios effect, suppression of the coffee stain effect and electrofreezing under an EW field, are also reviewed in Section 5. Finally, we discuss some difficult challenges both in theory and application and provide future research directions from our perspective.

\section{Theoretical basis}

The theoretical basis of EW was well established in the early 2000s. Lippmann-Young's equation, which associates CA changes with an electric field, is central to all mainstream physical principles in EW. Researchers from different fields have provided different interpretations of this equation. In this section, we will briefly introduce Lippmann-Young's equation from different points of view (Section 2.1). Additionally, the deviation of practical models from ideal models is discussed (Section 2.2).

\subsection{Lippmann-Young's equation}

Based on general Gibbs interfacial thermodynamics, an elegant derivation of eqn (1) can be obtained: ${ }^{35}$

$$
\gamma_{\mathrm{sl}}{ }^{\prime}=\gamma_{\mathrm{sl}}-\frac{1}{2} c V^{2}
$$

where $\gamma_{\mathrm{sl}}{ }^{\prime}$ and $\gamma_{\mathrm{sl}}$ are the solid/liquid interfacial tension with and without an electric field, respectively, $c$ is the differential capacity of the double layer between the liquid and electrode and $V$ is the applied voltage. This derivation indicates that the interfacial tension will decrease with the applied electric field. Combined with Young's equation:

$$
\cos \theta_{\mathrm{Y}}=\frac{\gamma_{\mathrm{sv}}-\gamma_{\mathrm{sl}}}{\gamma_{\mathrm{lv}}}
$$

where $\theta_{\mathrm{Y}}$ is Young's CA, $\gamma$ is the interfacial tension and $\mathrm{s}, \mathrm{l}$, and $\mathrm{v}$ are solid, liquid and vapour, respectively. Therefore, Young's equation under an electric field is

$$
\cos \theta=\frac{\gamma_{\mathrm{sv}}-\gamma_{\mathrm{sl}}{ }^{\prime}}{\gamma_{\mathrm{lv}}}=\frac{\gamma_{\mathrm{sv}}-\left(\gamma_{\mathrm{sl}}-\frac{1}{2} c V^{2}\right)}{\gamma_{\mathrm{lv}}}
$$

where $\theta$ is CA under the electric field; thus, we obtain Lippmann-Young's equation,

$$
\cos \theta=\cos \theta_{\mathrm{Y}}+\frac{1}{2} \frac{c V^{2}}{\gamma_{\mathrm{lv}}}
$$

This equation indicates the parabolic relationship between the cosine of real-time CA and the applied voltage. From the thermodynamics point of view, EW can be comprehended by the fact that the accumulation of same charges at the solidliquid interface increases the repulsive forces between the molecules on the surface of the droplet, thus decreasing the surface tension.

Another approach was published by B. Berge and co-workers. ${ }^{36}$ Their theoretical model is an ideal capacitor composed of a liquid droplet and planar electrode. In this case, EW is treated as a capacitor charging process. Because charge redistribution between the battery and capacitor decreases the energy of the system, the increased solid-liquid contact area caused by the 
CA change can increase the capacitance and thus redistribute the charge.

However, T. B. Jones et al. proposed that EW is an electromechanical process in nature ${ }^{24,37,38}$ which is gradually being accepted by more researchers. They modified Pellat's experiment to establish and analyse the theoretical model of a charged conductive droplet in an electric field. The net electrical body force on the droplet, named the Maxwell stress, results in the macroscopic EW behaviour. From this point of view, EW is an entirely electromechanical process and is irrespective of the CA, which was verified by F. Mugele and J. Buehrle. ${ }^{39}$ Based on their results, EW can only affect apparent CA, while the local CA near the three-phase contact line (TCL) is independent of the applied voltage at the scale of dielectric thickness. In other words, the microscopic CA is consistent with Young's CA. T. B. Jones also discussed the relationship between EW and DEP. ${ }^{24}$ Although they are essentially the same and often occur together, EW is the effect of an electric field upon the CA, while DEP refers to the liquid actuation caused by a net electromechanical force. This perspective provides a physical picture of CA reduction in mechanics and hence provides a theoretical basis for many applications based on liquid actuation.

\subsection{Various practical factors}

In almost all published papers, the parabolic relation of the applied voltage and the cosine of the measured CA partly deviate from Lippmann-Young's equation. ${ }^{40}$ The curve is shown to hold at low voltage; however, deviation occurs at high voltage. These deviations originate from the approximation of the ideal capacitor, which ignores various practical effects, including the fringe fields of the capacitor, the tip effect around the TCL, imperfect conduction of the electrolyte and electrification of the dielectric layer. Moreover, a phenomenon named contact angle saturation (CAS) is always found when exceeding the critical voltage. To date, the studies on the physical mechanism of CAS are far from conclusive; this is commonly considered to be one of the most important challenges in this field.

From the viewpoint of the electromechanical process, sufficient Maxwell stress can lead to complete spreading of droplets. In other words, according to eqn (5), CA can reach $0^{\circ}$ as long as the applied voltage is large enough. However, the effective voltage applied to the droplet is always lower than the voltage applied to the whole system. To determine the reason for this voltage loss, researchers have reported several hypotheses regarding CA saturation. As reported by A. G. Papathanasiou and A. G. Boudouvis, macroscopic electrostatic analysis showed that the electric field generated around TCL was ten times stronger than the surrounding uniform field. ${ }^{41}$ They hypothesised that charge is injected and trapped in the dielectric film, resulting in local dielectric breakdown near the TCL. The trapped charge in the dielectric film has no effect on EWOD, and the resulting charge shielding effect decreases the Maxwell stress. This trapped charge model was validated by analysing charge migration between the trapped charge states and liquid charge states in EW at lipid bilayers. ${ }^{42}$ Trapped charges decrease the Maxwell stress and eventually result in CAS. Although this mechanism may apply in some EWOD cases, trapped charge and local dielectric breakdown can be avoided by applying alternating voltages and using high dielectric-strength dielectric films. Another mechanism is electrostatic repulsion. A charged droplet on the electrode substrate usually generates a ring of satellite droplets around the mother droplet. ${ }^{36}$ The droplet becomes unstable under high voltage, and its CA decrease is prevented as a result of electrostatic repulsion. However, electrostatic repulsion decreases significantly when the conductivity of the droplets is approved.

Because CAS is a universal phenomenon in EWOD devices, a general mechanism that is irrespective of the materials and other experimental conditions is more convincing. The mechanism of ionization of the insulating surroundings was first proposed by B. Berge and co-workers. ${ }^{43}$ They observed luminescence caused by gas ionization at TCL and analysed the related electrical discharge. Charge leakage induces gas luminescence and thus decreases the driving force in EWOD. Molecular simulations validated that the apparent CA deviates from Lippmann-Young's equation when charges escape from the droplet and leak into its surrounding medium. ${ }^{44}$ For immiscible liquid surroundings, D. Andelman et al. ${ }^{45}$ predicted that ions could be injected from the aqueous droplet into the surrounding oil phase, resulting in CAS as well.

Finally, one study that must be mentioned is that by J. Heikenfeld et al., ${ }^{46}$ who experimentally validated that CAS was invariant with many factors in EWOD, including dielectric thickness, interfacial tension, $\mathrm{pH}$, ion type and size, solute/ solvent interaction, DC vs. AC, oil surface tension and dielectric type. Because the final saturated CA was basically around $60^{\circ}$ regardless of the abovementioned conditions, they believed that these experimental data refuted some existing hypotheses, including dielectric charging, surrounding charging and electrostatic repulsion. At least, they demonstrated that the existing hypotheses had many limitations, although this work did not unambiguously establish the cause of CAS. Additionally, these experimental data clearly indicate that saturation is a time-dependent phenomenon, and different physical mechanisms may play leading roles at different time scales during saturation. Therefore, pursuit of a universal theory for CAS continues.

\section{Electrodes and fluid systems in EWOD}

Diagrams of EWOD are shown in Fig. 2 (inside the circle), including a conductive droplet and an electrode covered with dielectric and hydrophobic layers. The CA of the droplet decreases with increasing electric field. In the case of a superhydrophobic system, the initial CA is larger than $150^{\circ}$ and decreases to $\sim 100^{\circ}$ under a certain electric field. EWOD with superhydrophobic electrodes shows robust performance, especially in reversibility and weak friction of the solid-liquid interface, both of which are important in droplet manipulation and practical applications (Section 4). 


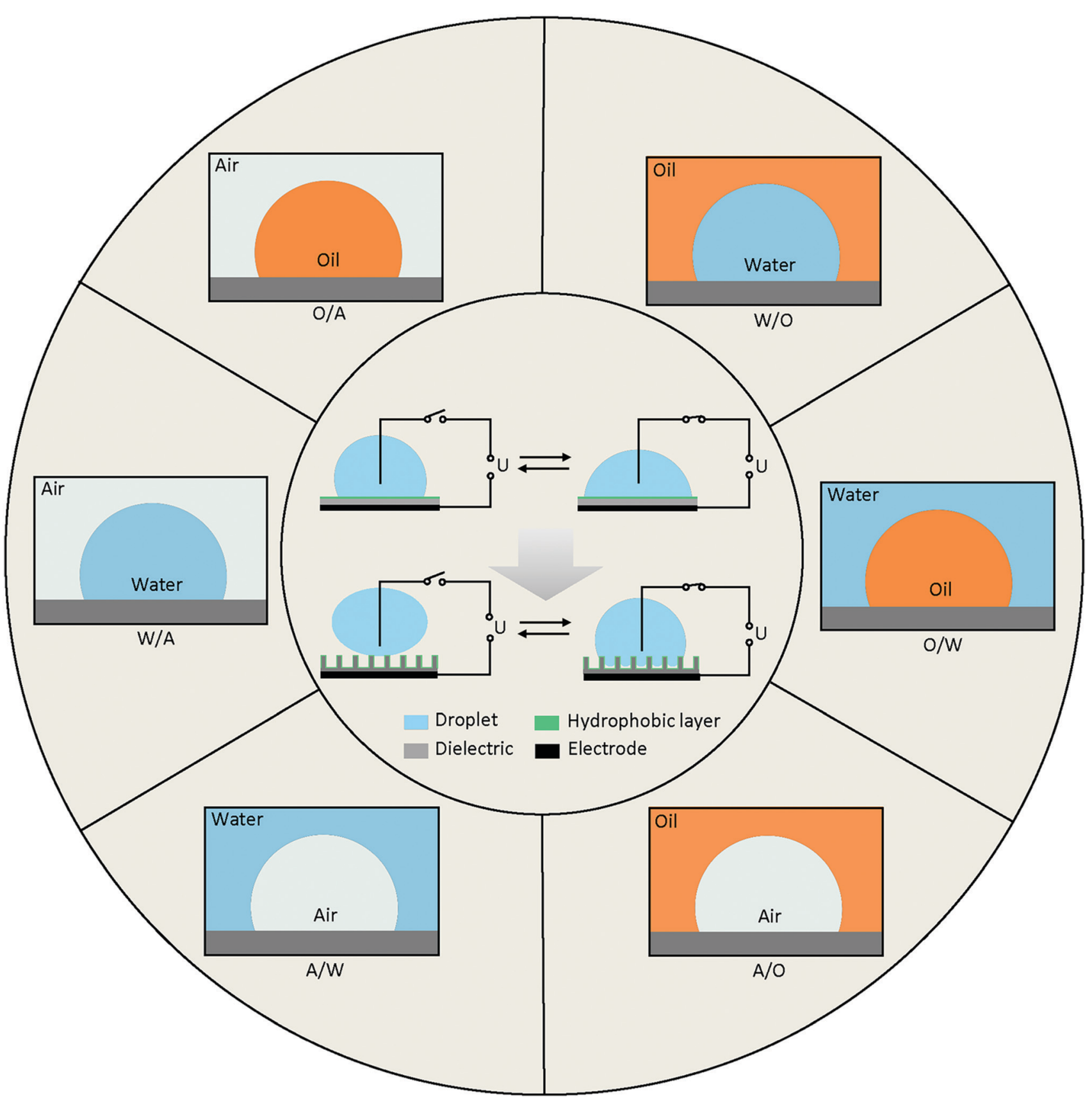

Fig. 2 EWOD from wetting to superwetting (inside the circle) with six basic fluid systems (outside the circle) based on three phases of water, oil and air, namely water in air (W/A), water in oil (W/O), oil in water (O/W), oil in air (O/A), air in water (A/W) and air in oil (A/O).

The CA change range is affected by various properties of the electrode and fluid system, which is one of the most important characteristics in EWOD. Over the past decades, innumerable materials with different functions were introduced in EWOD to enhance the performance of CA changes on demand. For example, superhydrophobic electrodes with micro- and nanostructured surfaces are used to reduce friction between the droplet and surface electrode. Meanwhile, reduction of the pinning force around TCL was demonstrated to be an excellent strategy for actuation of a large range of CA changes. Furthermore, diversified liquid systems composed of immiscible electrolytic solutions were introduced to achieve more robust EWOD and develop a series of applications. In this section, we will review the electrodes and fluid systems over the past decades to demonstrate their accomplishments and contributions in practical applications. The electrodes with various properties and surface morphologies are discussed in Section 3.1, and the fluid systems with multivariate components and surroundings are presented in Section 3.2.

\subsection{Electrodes in EWOD}

3.1.1. Electrodes with micro- and nanostructures. According to eqn (5), the initial WCA beyond $150^{\circ}$ of these special surfaces 
has great importance for droplet manipulation. Thus, the construction of a superhydrophobic electrode surface is very important for applications of EWOD. Inspired by nature, various superhydrophobic interfacial materials have been fabricated with the development of interface science and microand nanofabrication technology. ${ }^{47}$ Based on superhydrophobic electrodes, large CA changes can be realized in EWOD. As shown in Fig. 3a, there are two main methods to fabricate the superhydrophobic electrodes required for EWOD. One method is to cover a high-performance dielectric layer on a micro- and nanostructured conductor ( $\mu$-electrode), such as silicon nanowires ${ }^{48}$ and carbon nanotubes. ${ }^{49}$ Another method is to fabricate microand nanostructured dielectric layers, such as SU-8 photoresist ${ }^{50}$ and Parylene $\mathrm{C}^{51}$ on smooth electrodes. Furthermore, it is necessary to cover a low surface energy layer (e.g., fluoropolymer) on the dielectric layer in both methods. Among these, the $\mu$-electrode method is much simpler because the fabrication

a

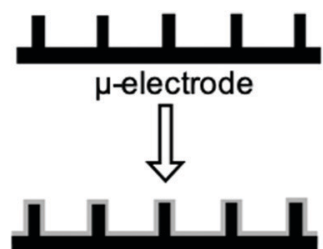

Dielectric layer coated<smiles>[C]=C=[CH]</smiles>

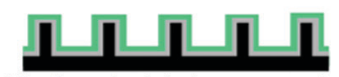

Hydrophobic layer coated

b
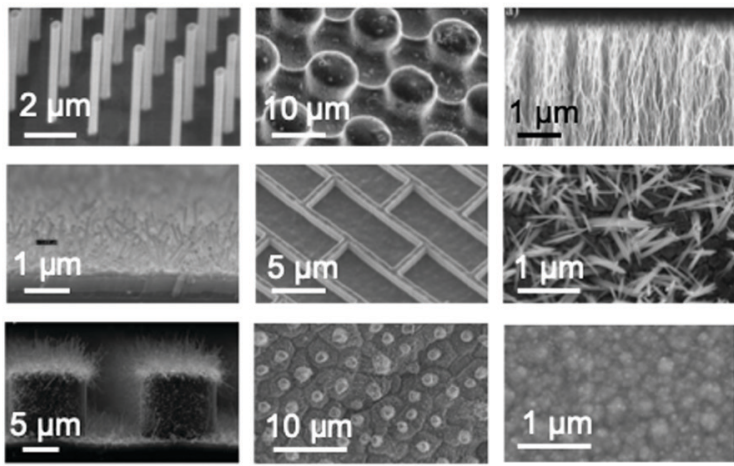

C
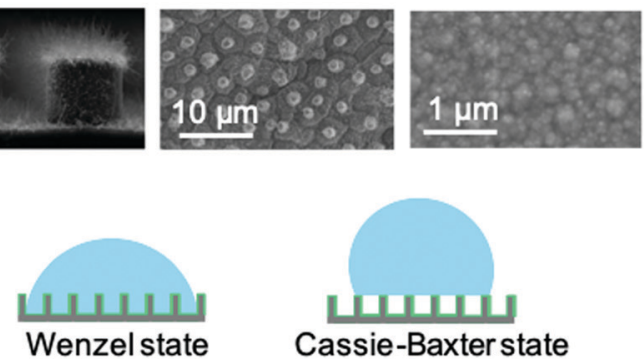

Fig. 3 Micro- and nanostructured electrodes. (a) Two methods to fabricate micro- and nanostructured electrodes. The left method involves covering a dielectric layer on a micro- or nanostructured conductor and the right method involves fabricating micro- and nanostructured dielectric layers on planar electrodes. (b) Various micro- and nanostructured electrodes have been reported, including Si nanopillars, SU- 8 microposts, aligned carbon nanofibres, Si nanowires, Si nanobricks, ZnO nanorods, Si nanowires on $\mathrm{Si}$ micropillars, lotus leaf structures and polypyrrole nano-protrusions, respectively, from left to right and top to bottom. (c) Two wetting states, named the Wenzel state and Cassie-Baxter state, respectively. technology of silicon and carbon is mature. An obvious inadequacy of this method is that the selectivity of the electrode materials is very limited.

Typical microstructured electrodes are shown in Fig. 3b, including Si nanopillars, ${ }^{26} \mathrm{SU}-8$ microposts, ${ }^{50}$ aligned carbon nanofibres, ${ }^{52} \mathrm{Si}$ nanowires, ${ }^{48} \mathrm{Si}$ nanobricks, ${ }^{53} \mathrm{ZnO}$ nanorods, ${ }^{54}$ Si nanowires ${ }^{48}$ on $\mathrm{Si}$ micropillars, ${ }^{55}$ lotus leaf structures ${ }^{56}$ and polypyrrole nano-protrusions. ${ }^{57}$ These micro- and nanostructures are of great significance to the research and application of materials with different dimensions. Although the surface morphologies are different, the CA change ranges on these electrodes are quite similar. As a driving electric field of tens of volts is added, the apparent CA will decrease from $>150^{\circ}$ to $\sim 100^{\circ}$. As shown in Fig. 3c, two models are used to analyse the wetting states on micro- and nanostructured surfaces, i.e., the Wenzel state and Cassie-Baxter state. For the Cassie-Baxter state, air pockets are trapped between droplet and surface structure, while in the Wenzel state, the droplet permeates into the surface structure. For example, T. N. Krupenkin et al. ${ }^{26}$ demonstrated that EWOD on micro- and nanostructured surfaces undergo a transition from the Cassie-Baxter model to the Wenzel model. During this transition, the energy dissipation originates from the propagation of liquid implemented in the micro- and nanostructures. As a result, the pinning force around TCL is so enormous that the transition becomes irreversible when the electric field is removed. ${ }^{53,58}$ From this point of view, two general methods can be used to reverse the wettability transition from the Wenzel state to the Cassie state, thereby providing additional energy to this system and minimizing undesirable energy dissipation.

Introducing additional energy is an effective method to realize the wettability transition from the Wenzel state to the Cassie-Baxter state. N. Krupenkin et al. ${ }^{53}$ realized reversibility of EWOD by momentarily increasing the temperature of the substrate surface. High temperature is conducive to increase the volume of trapped gas and vapor to reach the Cassie-Baxter state and hence promote the reversibility. Also, C. J. Kim et al. ${ }^{59}$ reported a method of electrolysis to generate gas from an electrode surface, inducing the Wenzel state to Cassie-Baxter state transition. Another reported method that promotes leaving the sticky Wenzel state is droplet vibration, which is accomplished by direct vertical vibration of the substrate ${ }^{60}$ and droplet oscillation (see Section 4.2).

Decreasing energy dissipation in the wetting process is another effective way to achieve a reversible wettability transition. During this process, surface engineering of the electrode is a necessary method to obtain a surface with low energy dissipation. The pinning force at this electrode surface can be effectively decreased. Additionally, EWOD of an aqueous droplet in an oil environment has been widely studied. The pinning force is significantly suppressed due to the lubricant effect of the oil film trapped between the electrode surface and droplet, which is further discussed in $\mathrm{W} / \mathrm{O}$ and $\mathrm{O} / \mathrm{W}$ systems (Section 3.2.2). It must be emphasized that energy dissipation in EWOD originates from the pinning force at the TCL, which plays a more important role in the reversibility of EWOD, as reviewed 
by A. G. Papathanasiou. ${ }^{61}$ For an EWOD system with a given surface chemistry and roughness, the surface tension and pinning force at the TCL are the key driving forces to actuate the droplet movement. From this viewpoint, A. G. Papathanasiou proposed more concrete alternatives to promote reversibility: (i) creating dual scale roughness with dense structures with low solid fractions to realize moderate energy loss from micro-Wenzel (but nano-Cassie) to micro-Cassie (also nano-Cassie), ${ }^{62}$ and (ii) using thick dielectrics to avoid high liquid profile curvature because decreasing the radius of curvature causes the liquid to substitute the air gaps between the asperities.

3.1.2. Electrodes with special functions. The selection of an electrode material depends on the application. For example, silicon is widely used in MEMS because of its mature micro-and nano-processing technology, and indium tin oxide (ITO) glass is essential for optical applications. In recent years, many novel electrodes with special functions have been reported. C. Kim et $a l^{25}$ first proposed optoelectrowetting (OEW) effects using flat electrodes with photoelectric conversion capability. Then, S. Park et al. ${ }^{63}$ and S. T. Wereley et al. ${ }^{64}$ used light to realize precise control of actuating picoliter droplets, which were introduced immediately in labs on chips to precisely control the droplet manipulation. Additionally, combined with special micro- and nano structures, our group ${ }^{30,65-68}$ proposed a photoelectric cooperative wetting (PCW) effect for light-operated permeation and patterning of droplets.

The pyroelectric effect has also been introduced in EW. Lithium niobate $\left(\mathrm{LiNbO}_{3}\right)$ is a colourless solid that is insoluble in water. It can generate a temporary voltage when it is heated or cooled. Based on the pyroelectric effect, P. Ferraro et al. ${ }^{69}$ designed a liquid micro-lens controlled by a temperature gradient. These results expanded the forms to activate EW instead of the single electric field. From our perspective, other stimuli, such as magnetic and mechanical fields, can also be utilized to activate EW; thus, it is applicable in more fields.

\subsection{Fluid systems in EWOD}

The fluid system is an important and decisive factor for achieving different EWOD performance. According to actual requirements, there are numerous combinations of fluid systems containing various components of droplet and surroundings. To simplify this, we provide an outline based on the different types of TCLS (Fig. 2), including water in air (W/A), water in oil (W/O), oil in water $(\mathrm{O} / \mathrm{W})$, oil in air $(\mathrm{O} / \mathrm{A})$, air in water $(\mathrm{A} / \mathrm{W})$ and air in oil $(\mathrm{A} / \mathrm{O})$.

Many of these systems have been studied, and a series of applications has been developed. For example, most digital microfluidics are based on W/A and O/A systems, and variablefocus microlenses always utilize $\mathrm{W} / \mathrm{O}$ and $\mathrm{O} / \mathrm{W}$ systems. $\mathrm{A} / \mathrm{W}$ and $\mathrm{A} / \mathrm{O}$ systems involve bubble and microcapsule manipulation, which is also a research focus in labs on chips (LOC).

3.2.1. W/A and O/A systems. The W/A system is the most common configuration in EWOD. WCA changes of about $30^{\circ}$ to $50^{\circ}$ can cause sufficient Laplace pressure to actuate a droplet. ${ }^{70}$ Based on this, many modes of droplet actuation have been developed, which will be discussed in detail in Section 4 . Additionally, most microfluidic devices are fabricated with
W/A systems because of its simple process and robust performance. $^{22,71-73}$ Also, for O/A systems, the actuation of oil droplets mainly depends on the DEP effect. Experimental studies on $\mathrm{O} / \mathrm{A}$ systems provide basic theory for actuating oil droplets and designing nonaqueous EWOD. R. L. Garrell ${ }^{74}$ et al. studied the actuation of dozens of common oils for nonaqueous microfluidics, and they listed various physical parameters of the tested liquids. Based on the large quantities of experimental data, they summarized a moving rule of liquid for molecular dipole moments greater than $0.9 \mathrm{D}$, dielectric constants greater than 3 and/or conductivities greater than $10^{-9} \mathrm{~S} \mathrm{~m}^{-1}$.

The influence of viscosity should also be considered in the actuation of oil. Existing research indicates that the viscosity only affects the magnitude of actuation and makes no difference in the principles of EW. Y. Fouillet et al. ${ }^{75}$ reported that viscosity mainly impacts the velocity of droplet actuation. However, the minimum voltage required for actuation is independent of the viscosity. They suggested that the velocity decreases with increasing viscosity mainly because of the friction at the TCL, and no further conclusions were made on this point. S. Lee et al. ${ }^{76}$ studied the influence of viscosity on oscillation. They demonstrated that the viscosity of the drop only affects the oscillation amplitude and not the resonance frequency. When the drop viscosity is beyond the critical value, drop oscillation is suppressed.

Ionic liquids (ILs) can be considered as another type of oil in O/A systems. The use of ILs as a novel class of electrolyte in EWOD was first reported by J. Ralston and co-workers. ${ }^{29}$ They demonstrated that imidazolium- and pyrrolidinium-based ILs show small ranges of CA change $\left(\sim 10\right.$ to $\left.20^{\circ}\right)$ on Teflon AF 1600. The relatively small CA change is mainly due to the small initial CA $\left(\sim 70^{\circ}\right)$. The maximum CA change of ILs under EWOD is about $50^{\circ} .{ }^{77}$ Also, asymmetric responses of positive and negative voltage are common in ILs; this may originate from the asymmetry of the cation and anion in ILs. Later, many EWOD behaviors of different types of ILs and relevant microfluidics devices were successively reported by researchers. ${ }^{77-79}$

3.2.2. $W / O$ and $O / W$ systems. $W / O$ and $O / W$ systems are models extracted from two immiscible liquids in EWOD; they can be further divided into two immiscible electrolytic solutions and two immiscible pure-dielectric liquids. For electrolytic solutions, the two liquid phases both contain certain electrolytes, which are preferentially soluble in water and nonpolar organic liquids. Also, transportation across the interface between two immiscible electrolytic solutions (ITIES) is blocked because of the large free energies of transfer. Under an electric field, a back-to-back electrical double layer is formed at the ITIES, resulting in a lower actuating voltage of hundreds of millivolts compared with 10 to $100 \mathrm{~V}$ in current EWOD devices. $^{80}$ This difference arises from the intensity of the electrical double layers. In typical systems, the electric field is evenly distributed across the insulating-liquid phase, resulting in considerable voltage losses in its bulk. Meanwhile, the electrical double layers at the ITIES localize potential drop crossing to only several nanometres. ${ }^{81}$ Applications of $\mathrm{O} / \mathrm{W}$ 
systems with electrical double layers at the ITIES mainly include analysis of adsorption characteristics, energetics of ion solvation and mesoscale interfacial morphology, ${ }^{82}$ which relates more to electrochemistry.

EWOD of two immiscible pure-dielectric liquids (or where at least one is dielectric) is the foundation of electrically controlled variable-focus microlenses and displays. A small droplet of insoluble oil is immersed in a conductive aqueous solution or vice versa. Under an electric field, the wettability of the electrode surface is significantly enhanced, modifying the morphology of the confined droplet and hence the focal length of the liquid lens. This concept is known as competitive electrowetting and was proposed by B. Janocha and co-workers. ${ }^{23}$

Compared with ambient air, the EWOD of aqueous droplets in an oil environment minimizes both evaporation and CA hysteresis, resulting in enhanced repeatability and reversibility. The CA hysteresis is very small $\left(<2^{\circ}\right)$ because the oil film trapped in the electrode surface plays a role in lubrication. ${ }^{83,84}$ H. Verheijen and M. Prins ${ }^{85}$ first reported reversible EW using an aqueous droplet on a Teflon AF 1600 hydrophobic layer, which is of great significance in commercializing EWOD based microlenses and displays. ${ }^{86-88}$ Additionally, W/O system-based droplet manipulation demonstrates faster velocities and lower actuating voltages compared to air environments. ${ }^{89}$

A special W/O system, named electrowetting on liquid-infused film (EWOLF), was proposed by Z. Wang and co-workers. ${ }^{90}$ As a dielectric layer, a liquid-infused film is achieved by locking a liquid lubricant (e.g. silicon oil) in a porous substrate. The pinning force at the contact line of the liquid-liquid interface is negligible; thus, this configuration leads to fast EWOD response with excellent reversibility compared with conventional EWOD on superhydrophobic electrodes. The transient response of EWOLF also demonstrated unexpected excellence, consuming only $45 \mathrm{~ms}$ to stabilize droplet oscillation compared with $500 \mathrm{~ms}$ of conventional EWOD. ${ }^{90}$ The suppression of droplet oscillation in the transient response is attributed to the viscous liquid-liquid interface. The is because additional wetting ridges around droplets can anchor contact lines and thus enhance dissipation of viscous oscillation energy. Subsequent research achieved low actuating voltages ${ }^{91}$ (decrease from $500 \mathrm{~V}$ to $5 \mathrm{~V}$ ) and digital microfluidics with fast droplet transport ( $85 \mathrm{~mm} \mathrm{~s}^{-1}$ ) and stable repeatability (>5000 times), ${ }^{92}$ showing great performance improvement compared with typical EWOD devices.

For $\mathrm{O} / \mathrm{W}$ systems, an oil droplet immersed in water weakly adheres to a hydrophobic electrode surface. Based on this, our group realized movement of an oil droplet on a gradient pore polystyrene substrate because the adhesion between the oil droplet and substrate could be efficiently decreased in the $\mathrm{O} / \mathrm{W}$ system. ${ }^{33,93}$ Another peculiar phenomenon in $\mathrm{O} / \mathrm{W}$ systems is detachment of the oil droplet from the electrode surface. A typical $\mathrm{O} / \mathrm{W}$ system is formed by an oil droplet adhered on a hydrophobic polymer surface with surrounding water. When the electric field is applied, the CA of the oil droplet increases due to the competitive effect of the surrounding water, ${ }^{23}$ ultimately leading to oil droplet detachment. The critical conditions for detachment depend on the droplet volume, viscosity and applied voltage, as proposed by J. Hong and S. Lee. ${ }^{94}$ They also demonstrated three-dimensional digital microfluidic (3D-DMF) platforms with the aid of vertical movement of the oil droplet, which is of great importance in cell culture, electronic cooling, and sampling of microparticles.

3.2.3. $\mathrm{A} / \mathrm{W}$ and $\mathrm{A} / \mathrm{O}$ systems. $\mathrm{A} / \mathrm{W}$ and $\mathrm{A} / \mathrm{O}$ systems focus on the manipulation technology of bubbles under an electric field. Because bubbles are common in everyday life, they are long-standing research topics in physics, chemistry, medicine, engineering, etc. Bubble manipulation by EWOD was first proposed by S. Cho and co-workers. ${ }^{27}$ Briefly, the CA of a capped air bubble in a water environment was measured under an electric field and was confirmed to be in good agreement with Lippmann-Young's equation. Also, they demonstrated the basic operations of bubble manipulation in DMF with high degrees of freedom and precision, including transporting, splitting, merging and eliminating. They also demonstrated that bubbles can be used as microcarriers to transport micro objects by directly contacting the objects. This group further studied micro-object manipulation in a variety of objects, ${ }^{95,96}$ including glass beads $(80 \mu \mathrm{m})$, polystyrene beads $(100 \mu \mathrm{m})$, a fish egg $(\sim 1 \mathrm{~mm})$ and a live water flea $(\sim 1 \mathrm{~mm})$. This approach enriches the target in DMF and thus broadens the applications of integrated lab chip microsystems.

Bubbles will retract under an electric field due to a decrease of the ambient CA. Consequently, adhesion between the bubbles and substrate decreases as a result of the decreased conformal contact. When the adhesion force is decreased below the buoyancy of the bubbles, detachment can be observed. ${ }^{93,97}$ Moreover, manipulation of bubbles is significant in heat transfer. By controlling bubble detachment, it is possible to adjust the temperature or steam generation in the process of heat transfer. E. Wang et $a .^{98}$ reported a switch of heat transfer through in situ manipulation of bubbles. Under the influence of an electric field, bubble nucleation is significantly enhanced; hence, the heat transfer coefficient increases.

\section{Droplet manipulation}

Droplet manipulation is the foundation of all applications of EWOD. Physical descriptions of droplet movement through asymmetric EWOD actuation can be interpreted from different perspectives, including asymmetric electrostatic energy, surface tension and hydrostatic pressure, as summarized by W. Nelson and C. J. Kim. ${ }^{70}$ These descriptions are not contradictory. In fact, they enrich the understanding of EWOD-driven droplet movement from different aspects.

As an illustration, Fig. 4 shows a cross section of a droplet in a typical EWOD device. The CA in the original state is assumed as $90^{\circ}$ to simplify the analysis and focus more on the active region. When the lower right electrode is activated and all other electrodes are grounded, the droplet moves to the right as its CA changes to $\theta_{\mathrm{V}}$. It should be pointed out in particular that the Young's angle is maintained, as shown in the enlarged figures. 

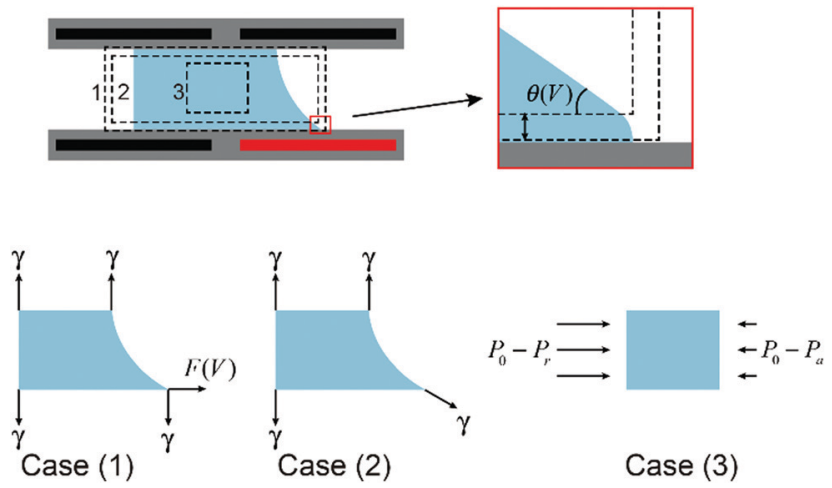

Fig. 4 Cross section of a droplet in a typical EWOD device with force analysis from different perspectives.

Young's angle is a microscopic expression of the distance of $\chi$ from an electrode surface, as discussed previously in Section 2.1. It has been proven that EWOD can only affect the apparent CA, whereas the microscopic CA near the TCL is independent of the applied voltage. ${ }^{39}$ From the perspectives of micro-angle (or Young's angle) and macro-angle (or apparent angle), force analysis can be divided into case (1) and case (2), respectively. As shown in the figure, in case (1), the net force acting on the droplet in the horizontal direction is

$$
F=F(V)
$$

where $F(V)$ is the Maxwell pressure in the horizontal direction. ${ }^{24}$ However, case (2) is located farther than $\chi$ away from the electrode surface. The Maxwell pressure in this scale is significantly decreased to negligible levels. ${ }^{99}$ Thus, the net force in case (2) in the horizontal direction is

$$
F=\gamma \cos \theta_{V}
$$

where $\gamma$ is the surface tension of the liquid-fluid interface. Case (3) encloses a liquid volume which is irrespective of the electric field and surface tension. The driving force in case (3) is the unbalanced hydrostatic pressure, as shown in the figure. The pressure on the advancing side is $P_{0}-P_{\mathrm{a}}$, where $P_{0}$ is the atmospheric pressure and $P_{\mathrm{a}}$ is the Laplace pressure of the advancing side. Similarly, the pressure on the receding side is $P_{0}-P_{\mathrm{r}}$. Thus, the net force in case (3) is

$$
F=\left[\left(P_{0}-P_{\mathrm{r}}\right)-\left(P_{0}-P_{\mathrm{a}}\right)\right] h=\left(P_{\mathrm{a}}-P_{\mathrm{r}}\right) h
$$

where $h$ is the height of the droplet cross section. Because $P_{\mathrm{a}}$ is always larger than $P_{\mathrm{r}}$, this net force is invariably applied on the droplet towards the activated EWOD side. As expected, the above three expressions of the EWOD driving force can be interchanged; that is,

$$
F=F(V)=\gamma \cos \theta_{\mathrm{V}}=\left(P_{\mathrm{a}}-P_{\mathrm{r}}\right) h
$$

Based on these three equations, W. Nelson and C. J. Kim demonstrated that different forces may coexist in EWOD. ${ }^{70}$ From the microscopic perspective, an electrostatic force applied on the TCL pulls the droplet towards the activated EWOD side (case 1). As a result, the apparent CA decreases; this changes the liquid-fluid surface tension applied on the droplet. The unbalanced surface tension force tends to pull the droplet forward (case 2). Accompanied by a CA decrease, the curvature of the liquid-fluid interface decreases; hence, the Laplace pressure increases on the advancing side. As a consequence, the hydrostatic pressure on the receding side is larger than that on the advancing side, pulling the droplet towards the activated side (case 3). According to a certain application, one of these methods may be more favorable than others. For example, the electrostatic method is more suitable for the physical nature of the simple EWOD model (e.g., analysis of CA saturation). For more complicated models, such as $\mathrm{O} / \mathrm{W}$ and $\mathrm{O} / \mathrm{W}$ systems, the surface tension method is preferred because the more macroscopic perspective is advantageous to the overall analysis. Also, in order to understand more concrete issues (e.g. the flow distribution in EWOD-driven droplets), the hydrostatic method is probably the most useful.

Based on the movement of droplets under EWOD, we reviewed various modes of droplet manipulation, such as transfer, oscillation, capillary bridge, permeation and bouncing. The basic development overviews and potential applications of each mode are summarized in this section.

\subsection{Transfer}

Droplet transfer is the most typical and common manipulation mode in many EWOD devices. The earliest proposal of droplet transfer in EW dates back to 1990. E. Matsumoto et al. ${ }^{100}$ proposed a model describing liquid flow in a small channel. As their simulation results demonstrated, EW was a viable strategy for microactuation. For a $10 \mu \mathrm{m}$ radius channel, the pressure generated by EW can reach $0.01 \mathrm{MPa}$, which is comparable to a micropump. Subsequently, M. Pollack and R. Fair et $a .^{22}$ reported the transfer of discrete microdroplets based on EW. As shown in Fig. 5a, two planar electrodes were fabricated on glass, where the bottom electrode consisted of a linear array of spaced electrodes. When a voltage of 40 to $80 \mathrm{~V}$ was applied, saline droplets could be successively transferred at average velocities of $30 \mathrm{~mm} \mathrm{~s}^{-1}$. These studies laid the foundation for subsequent microfluidic research. More recently, a new strategy to manipulate underwater oil droplets for unidirectional motion was demonstrated by our group. ${ }^{33}$ The effects of EW and surface gradient wettability cooperated to realize continuous oil droplet transfer.

The aforementioned sandwich structure of electrodes and electrolyte is complex and results in a very large frictional force that restricts droplet transfer. To address these problems, U. Yi and C. Kim ${ }^{101}$ studied EWOD actuation on coplanar electrodes with no cover plate (Fig. 5b). They demonstrated basic droplet manipulation techniques, including moving, cutting and merging of $0.1 \mu \mathrm{L}$ droplets on this coplanar electrode. More sensing mechanisms can be introduced in this coplanar EWOD configuration and thus increase its flexibility for various applications.

Other special behaviours of droplet transport are sliding, climbing and uphilling. J. Kim and M. Kaviany ${ }^{102}$ studied the sliding behaviour of a droplet adhered on an inclined substrate (Fig. 5b). 
a

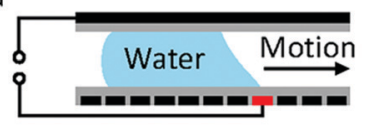

C

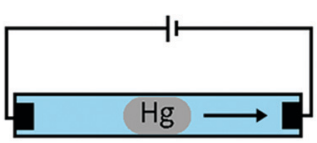

b

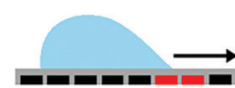

Translation

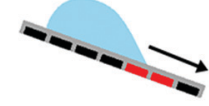

Sliding

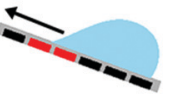

Climbing

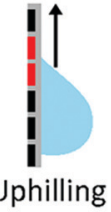

- Electrode - Active electrode
Fig. 5 Droplet transfer. (a) Water droplet transfer between two parallel electrode plates. (b) Water droplet sliding, climbing and uphilling on a coplanar electrode. (c) A mercury droplet immersed in saline is transferred towards the cathode.

For a sufficiently small droplet volume (Bond number less than 0.8 ), actuation is difficult because the effect of EW is limited. For a sufficiently large droplet (Bond number greater than 2.5), gravity dominates, and the droplet can slide spontaneously. In the Bond number region between 0.8 and 2.5 , the droplet adheres on the inclined surface at the start state and slides down when an appropriate voltage is applied. According to theoretical simulations, EW introduces a radial electrostatic force around the TCL, breaking the balance between surface tension and friction. As a result, the droplet can slide down when an appropriate voltage is applied. Droplet climbing on a inclined substrate and even uphilling on a vertical substrate were studied by A. K. Das and co-workers. ${ }^{103-106}$ They demonstrated that the driving force caused by EW could overcome gravity; this droplet manipulation can be utilized in integrated microfluidic analysis systems.

J. Lee and C. $\mathrm{Kim}^{107}$ explored a so-called continuous EW phenomenon for MEMS. A mercury droplet immersed in saline electrolyte can move towards a cathode (Fig. 5c) as a result of asymmetric polarization of the mercury droplet. With an applied voltage of only $2.8 \mathrm{~V}$, the transfer speed of a mercury droplet in this system could reach $40 \mathrm{~mm} \mathrm{~s}^{-1}$. Then, Wan et al. ${ }^{108}$ studied the fundamental EWOD behaviours of mercury. On a parylene film, a mercury droplet underwent CA changes of $74^{\circ}$ (from $141^{\circ}$ to $67^{\circ}$ ) with good reversibility under an electric field of $25 \mathrm{~V}$. Then, researchers focused on Galinstan, a nontoxic liquid-metal alloy. M. Dickey et al. ${ }^{109}$ demonstrated movement of a Galinstan liquid droplet inside a capillary wall utilizing a continuous EW effect. Recently, many applications based on EWOD of Galinstan have been reported, including a mechanical pump system, ${ }^{110}$ an integrated liquid cooling system $^{111}$ and a controlled electrical switch. ${ }^{112}$

\subsection{Oscillation}

When an AC electric field is applied, the actuation behaviour of the liquid droplet changes. Under low frequency EWOD, the droplet oscillates with variable voltage. Droplet oscillation under an AC electric field was first reported by M. Gunji and M. Washizu. ${ }^{113}$ They observed a transformation between the elongation and restored states of a saline droplet under an
AC electric field. Then, J. D. Sterling et $a .^{28}$ observed shape oscillations of droplets on a planar solid surface. With electric fields of $60 \mathrm{~V}$ and $180 \mathrm{~Hz}$, droplet oscillation was observed (Fig. 6a). Droplet oscillations were studied systematically by K. Kang et al. ${ }^{114}$ They demonstrated that excitation of droplets into various modes of oscillation depends significantly on the input frequency. As shown in Fig. 6b, the droplets oscillated at a certain input frequency, and the pattern of oscillation varied with different resonance frequencies. This study is significant for EW-based variable-focus microlenses and displays. Further developments should investigate the unstable nature of EW, which is important in EW-based liquid lenses and reflective displays.

Meanwhile, for sufficiently high frequency EWOD, circulatory flow inside the droplet was observed by K. H. Kang and co-workers. ${ }^{115}$ In the frequency range of $10 \mathrm{~Hz}$ to $15 \mathrm{kHz}$, they observed rapid flow. Also, in the frequency range of about 35 to $256 \mathrm{kHz}$, a distinct type of flow was observed which was also significantly affected by the electrolyte concentration (Fig. 6c). The driving mechanism of these hydrodynamic flows was provided by F. Mugele and co-workers. ${ }^{116}$ At this high frequency, energy dissipation of the electric field in the bulk liquid cannot be ignored because the Joule heating effect leads to a temperature increase of several degrees. As a result, the temperature increase leads to gradient distributions of conductivity and permittivity; thus, an applied electric field can induce hydrodynamic flow.

\subsection{Capillary bridge}

The study of capillary bridges caused by EWOD was first reported by F. Mugele and co-workers. ${ }^{117}$ As shown in Fig. 7a, two droplets formed a capillary bridge between two parallel electrodes. Under an electric field, both droplets tended to spread on the electrode; thus, the height of each droplet decreased. As a result, the droplet state was formed when it decreased to a critical height.

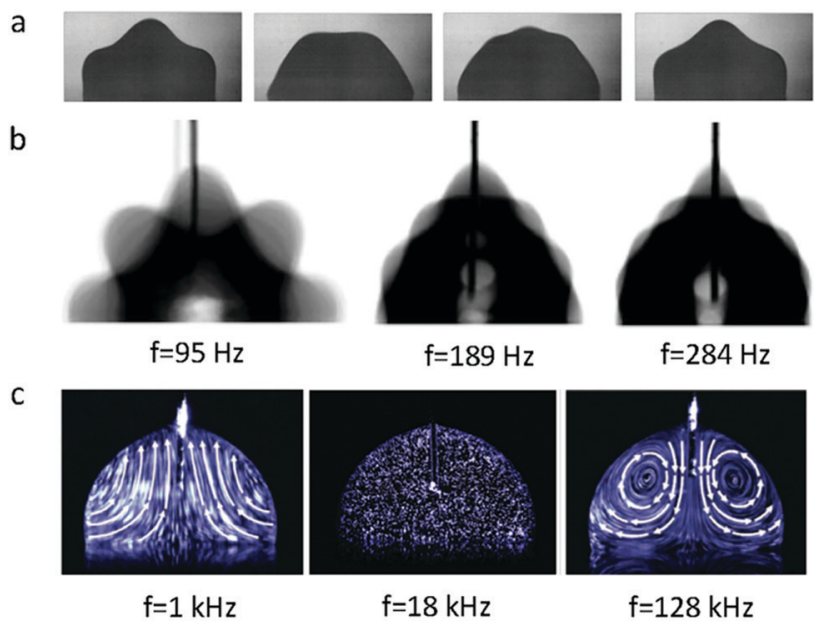

Fig. 6 Droplet oscillation under an AC electric field. (a) Oscillations under $60 \mathrm{~V}$ and $180 \mathrm{~Hz}$. Snapshots are $1 \mathrm{~ms}$ apart. (b) Various modes of oscillation under different resonance frequencies. (c) Rapid hydrodynamic flow was observed with $f=1 \mathrm{kHz}$. No hydrodynamic flow occurred with $f=18 \mathrm{kHz}$, and a distinct type of flow was observed when $f=128 \mathrm{kHz}$. 
a
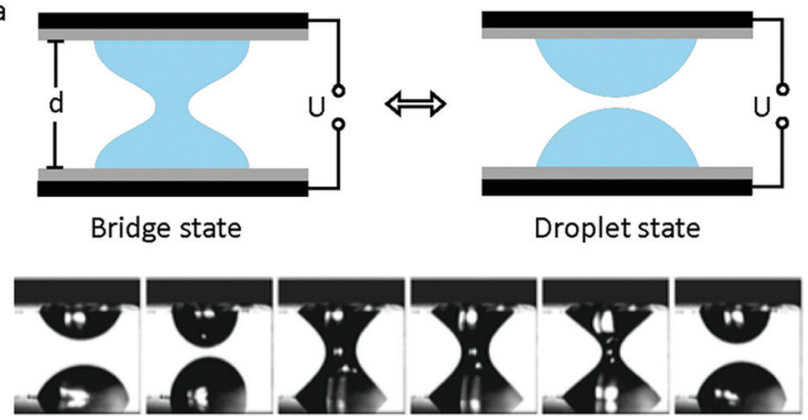

b

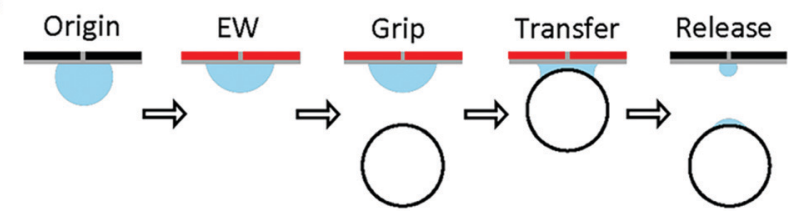

Fig. 7 A capillary bridge. (a) Droplets oscillate periodically between the droplet state and capillary state. The top is a diagram of the capillary bridge and the bottom is the captured experimental data. (b) A diagram of a microgripper. A glass bead is lifted up and released with the application of an electric field.

For an appropriate separation distance of the electrode, the system can oscillate periodically between the droplet state and capillary state. Additionally, the relationship among the droplet morphology, electrode separation and applied voltage was analysed. ${ }^{118}$ For different combinations of control parameters, three different states of the system existed, including stationary capillary bridges, stationary separated droplets and periodic oscillations.

This oscillation can promote the mixing performance of droplets on a millimetre scale. Compared to diffusion, the mixing speed of the oscillation system increases by 2 orders of magnitude. ${ }^{119}$ Moreover, Vasudev et al. ${ }^{120-122}$ proposed a microgripper based on manipulation of a liquid bridge (Fig. 7b), where the planar electrode was initially hydrophobic (CA around $120^{\circ}$ ). When an electric field was applied, the electrode became hydrophilic (CA less than $90^{\circ}$ ) and then approached a glass bead to form a liquid bridge. The bead was lifted up and transferred. When the applied voltage was removed, this microgripper released the glass bead instantaneously. The mechanism of this microgripper is based on the dynamic regulation of surface tension and capillary forces between the planar electrode and the object. Additionally, substitution of ionic liquids for water enabled this microgripper to function in high temperature and vacuum environments. ${ }^{123}$ Subsequently, Zhao et al. ${ }^{124}$ reported that adhesion between the droplet and superhydrophobic $\mathrm{MnO}_{2}$ nanotube arrays could be controlled by the polarity of the applied electric field. They demonstrated controllable liquid transportation between the upper and lower electrode plates. These systems have great prospects in the fields of micro-assembly and fabrication.

\subsection{Permeation}

Recently, an interesting phenomenon in which a droplet permeates into a superhydrophobic electrode triggered by an
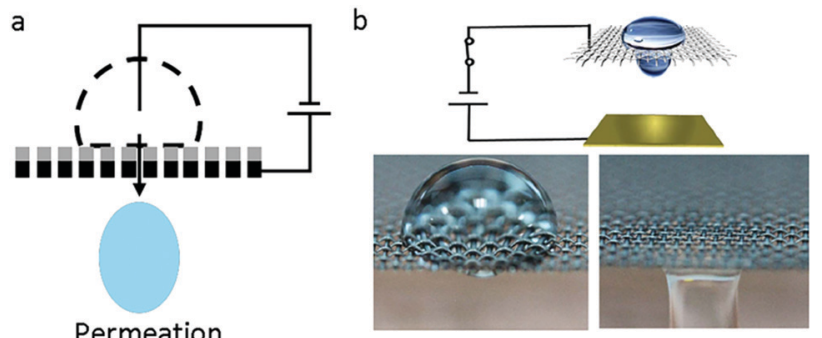

Fig. 8 Droplet permeation. (a) A diagram of droplet permeation. (b) Process of droplet permeation on a graphene-coated nickel mesh with an applied electric field.

electric field had aroused wide attention. V. Lifton et al. ${ }^{125}$ first reported an electrically tunable nanomesh with controllable droplet permeability based on EWOD. In the absence of voltage, the electrolyte formed a Cassie-Baxter state on a superhydrophobic membrane-filter, as shown in Fig. 8a. Upon applied voltage, the nanomesh became hydrophilic; hence, the liquid permeated it and then contacted the hydrophilic collector. Additionally, they proposed the application of a smart microbattery. ${ }^{126}$ In this smart microbattery, the electrochemical reaction is controlled by a tunable membrane separating the liquid electrolytes and solid electrodes. When this membrane is triggered by an electric field, electrolytes permeate through the electrodes and electricity is generated. The separation of electrolytes and electrodes has importance in prolonging service life because the active components in this battery are kept apart.

Our group $^{30,66}$ reported a liquid printing method based on the electric-triggered permeation of pigment droplets, realizing smart printing with controllable patterns. S. Nugen et al. ${ }^{127}$ utilized this electric-triggered permeation to control the rate of fluid flow on paper-fluidic sensors. Also, K. Oh et al. ${ }^{128}$ performed a comprehensive study on the flow control technique. They used graphene-coated nickel mesh as the flow-control membrane (Fig. 8b). The transition between repellency and permeability of the liquid droplet was controlled by an electric-responsive membrane. Furthermore, oil-water mixtures, including oil-inwater and water-in-oil emulsions, can be efficiently ( $\geq 99.9 \%)$ separated on demand based on the difference of oil and water permeability. ${ }^{129-131}$ These new strategies of EW-induced oil/water separation would be significant for smart filtration, microreaction, microfluidic systems, etc.

\subsection{Bouncing}

Another interesting behavior of a droplet in EWOD is bouncing on the substrate. Droplet bouncing was first reported by K. Kang and co-workers. ${ }^{114}$ When observing the oscillation of a droplet under AC electric field, they found that the droplet tended to bounce away from the substrate. Based on this, they performed systematic research on the droplet bouncing, utilizing a superhydrophobic substrate with a needle electrode above it (Fig. 9a). ${ }^{132,133}$ As shown in Fig. 9b, a saline droplet with a volume of $8 \mu \mathrm{L}$ bounced on a superhydrophobic substrate with an electric field of $100 \mathrm{~V}_{\mathrm{rms}}$ and 30 to $31 \mathrm{~Hz}$. In this configuration, the shape deformation and bouncing of the droplet significantly depend on 


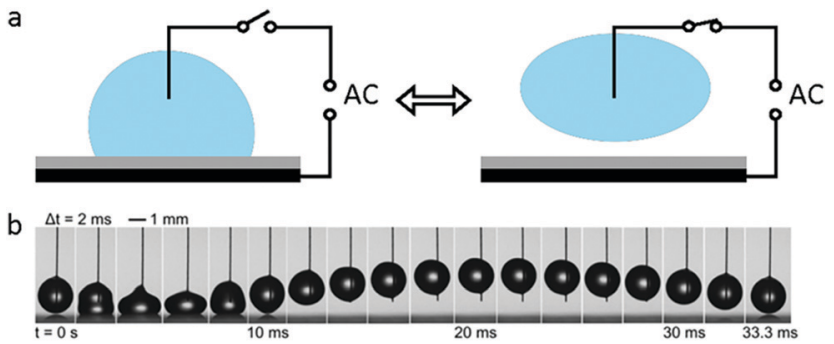

Fig. 9 Droplet bouncing. (a) A diagram of droplet bouncing under electric field. (b) A saline droplet with a volume of $8 \mu \mathrm{L}$ bouncing on a superhydrophobic substrate with an AC electric field of $100 \mathrm{~V}$ and 30 to $31 \mathrm{~Hz}$.

the AC frequency because droplet bouncing only occurs in very narrow frequency ranges. All these results indicate that droplet bouncing is dominated by resonance. They also studied the detailed bouncing mechanisms with regard to the jumping height and the energy conversion. ${ }^{133}$ They proposed that the work done by the applied electric field could stretch the droplet to store energy, and bouncing would occur when the electric field was removed. Based on the bouncing behavior, they demonstrated a 3D droplet digital microfluidic system in which a sessile droplet could be transported away from surfaces for further applications. This work provides conditions to study transporting droplets with antigravity and lays the foundation for more methods of droplet manipulation.

\section{Other special phenomena induced by EW}

As an efficient method to regulate solid-liquid interfacial properties, EW induces many special phenomena and interesting devices in some fields related to dynamic controllable wettability. For example, Mugele et al..$^{134}$ demonstrated regular coalescence of a droplet array condensing onto a flat hydrophobic electrode, which is due to the enhanced coalescence of droplets caused by structured electrodes. Furthermore, Chen et al. ${ }^{135}$ designed a quadrupedal microrobot that could easily sink and rise to the water surface based on four regulatable EW pads. In this section, we summarize other special phenomena relating to EW that have emerged in recent years in the hope of providing a more comprehensive understanding of EW.

\subsection{Controllable Cheerios effect}

The so-called "Cheerios effect" was first proposed by D. Vella and L. Mahadevan. ${ }^{136}$ They observed that breakfast cereals floating in milk always clump together or stick to the walls of the breakfast bowl. The Cheerios effect is a common phenomenon in which small floaters are either repelled or attracted by the sidewall of the container, depending on the combined effects of capillary interaction and buoyancy. ${ }^{137-139}$ Because buoyancy is related to the inherent properties of floaters, the regulation of capillary interaction is vital to control the Cheerios effect. This capillary force is highly dependent on the wettability of the sidewalls and floaters. Thus, repulsion or attraction between the floaters and the sidewall is meditated by CA changes. S. Cho et al. ${ }^{140}$ implemented EWOD to regulate the CA of a sidewall which was composed of a conductor and dielectric layer. Therefore, the interaction forces between the floaters and sidewall switched easily between repulsion and attraction. Based on this, N. Crane et al. ${ }^{141}$ reported accurate manipulation of floaters, including position, alignment, assembly and transport, demonstrating an alternative method for positioning and assembly.

\subsection{Suppressing the coffee stain effect}

The coffee stain effect originates from the differential evaporation rates between the edge and the interior of a droplet. ${ }^{142}$ The influence of EW on the coffee stain effect was studied by F. Mugele et al. ${ }^{143}$ They found that EW efficiently suppressed coffee stains of both colloidal particles and DNA solutions in an AC electric field. The mechanism of suppression caused by EW is based on the decrease of the pinning force at the TCL and enhancement of the internal mixing speed. The combination of these two effects prevents the accumulation of solutes around the TCL. These results have significance in improving detection sensitivity in the field of microfluidic technology, such as micro-detection and micro-reaction systems.

\subsection{Electrofreezing under EW}

Electrofreezing is the electrically induced freezing of supercooled water. Electrofreezing under EW studies the behavior of ice nucleation in water droplets in the $\mathrm{EW}$ configuration. V. Bahadur et al. ${ }^{144}$ and R. Shaw et al. ${ }^{145}$ demonstrated that the freezing temperatures of water droplets increased in an applied electric field. Also, the increase of the freezing temperature became saturated at high voltage. These results led to a deeper understanding of the mechanism of electrofreezing, where nucleation is primarily affected by the TCL instead of a previously recognized solid-liquid interface. This is because a hydraulic disturbance in supercooled water caused by EW can enhance crystallization. From our perspective, electrofreezing has potential for rapid advancement in the field of regulating liquid-solid phase changes.

\section{Conclusion and outlook}

In order to give a clear picture of the development of droplet manipulation in $\mathrm{EW}$, recent progress is summarized in this review. We provide an overview of the mainstream physical principles, various electrodes and fluid systems in EWOD, different droplet manipulation techniques and several special phenomena induced by EW. Additionally, the following challenges are worthy of further discussion for future study directions.

(1) Currently, the effect of the electric field on surface tension is difficult to measure directly. The alternative method is to characterize changes in the CA to indirectly test the surface tension. This method is not particularly convincing because the apparent CA does not fully represent microscale interfacial tension. Disclosing the mechanism of the effect of the electric field on interfacial tension will provide a deeper understanding 
of EW and may bring about a technological revolution in this field.

(2) The use of EW to dynamically control wettability can be complemented and perfected by theoretical construction of dynamic wetting.

(3) Developing multidimensional and multifunctional electrodes for new EWOD systems with enhanced reversibility and stability as well as multi-field cooperation will extend the fundamental research and applications of EW.

\section{Conflicts of interest}

There are no conflicts to declare.

\section{Acknowledgements}

This work was financially supported by the National Natural Science Foundation of China (Grant No. 21671012 and 61762034) and the Natural Science Foundation of Beijing Municipality (2172033).

\section{References}

1 K. Dholakia and T. Čižmár, Nat. Photonics, 2011, 5, 335-342.

2 S.-Y. Park and P.-Y. Chiou, Adv. OptoElectron., 2011, 2011, 909174.

3 J. R. Dorvee, A. M. Derfus, S. N. Bhatia and M. J. Sailor, Nat. Mater., 2004, 3, 896-899.

4 D. Tian, N. Zhang, X. Zheng, G. Hou, Y. Tian, Y. Du, L. Jiang and S. X. Dou, ACS Nano, 2016, 10, 6220-6226.

5 T. Franke, A. R. Abate, D. A. Weitz and A. Wixforth, Lab Chip, 2009, 9, 2625-2627.

6 Z. Wang and J. Zhe, Lab Chip, 2011, 11, 1280-1285.

7 J. Z. Chen, S. M. Troian, A. A. Darhuber and S. Wagner, J. Appl. Phys., 2005, 97, 014906.

8 E. F. Greco and R. O. Grigoriev, Phys. Fluids, 2009, 21, 042105.

9 G. Lippmann, Ann. Chim. Phys., 1875, 5, 494-549.

10 H. Pellat, C. R. Acad. Sci. Paris, 1895, 119, 691-694.

11 A. Frumkin, A. Gorodetskaya, B. Kabanov and N. Nekrasov, Phys. Z. Sowjetunion, 1932, 1, 255-284.

12 A. Frumkin and C. Double, Actual. Sci. Ind., 1936, 373, $5-36$.

13 A. Frumkin and A. Gorodetzkaja, Acta Physicochim. URSS, 1938, 9, 327-340.

14 G. Beni and S. Hackwood, Appl. Phys. Lett., 1981, 38, 207-209.

15 G. Beni and M. Tenan, J. Appl. Phys., 1981, 52, 6011-6015. 16 J. H. Jackel and S. Beni, Appl. Phys. Lett., 1982, 40, 4-5.

17 J. L. Jackel, S. Hackwood, J. J. Veselka and G. Beni, Appl. Opt., 1983, 22, 1765-1770.

18 J. A. M. Sondag-Huethorst and L. G. J. Fokkink, J. Electroanal. Chem., 1994, 367, 49-57.

19 J. A. M. Sondaghuethorst and L. G. J. Fokkink, Langmuir, 1994, 10, 4380-4387.
20 B. Berge, C. R. Acad. Sci. Paris Ser. II, 1993, 317, 157-163.

21 B. Berge and J. Peseux, Eur. Phys. J. E: Soft Matter Biol. Phys., 2000, 3, 159-163.

22 M. G. Pollack, R. B. Fair and A. D. Shenderov, Appl. Phys. Lett., 2000, 77, 1725-1726.

23 B. Janocha, H. Bauser, C. Oehr, H. Brunner and W. Gopel, Langmuir, 2000, 16, 3349-3354.

24 T. B. Jones, Langmuir, 2002, 18, 4437-4443.

25 P. Y. Chiou, H. Moon, H. Toshiyoshi, C. J. Kim and M. C. Wu, Sens. Actuators, A, 2003, 104, 222-228.

26 T. N. Krupenkin, J. A. Taylor, T. M. Schneider and S. Yang, Langmuir, 2004, 20, 3824-3827.

27 Y. Zhao and S. K. Cho, Micro bubble manipulation towards single cell handling tool, IEEE, 2006.

28 R. Miraghaie, J. D. Sterling and A. Nadim, Technical Proceedings of the 2006 NSTI Nanotechnology Conference and Trade Show, 2006, vol. 2, pp. 610-613.

29 S. Millefiorini, A. H. Tkaczyk, R. Sedev, J. Efthimiadis and J. Ralston, J. Am. Chem. Soc., 2006, 128, 3098-3101.

30 D. Tian, Q. Chen, F.-Q. Nie, J. Xu, Y. Song and L. Jiang, Adv. Mater., 2009, 21, 3744-3749.

31 T. Krupenkin and J. A. Taylor, Nat. Commun., 2011, 2, 448.

32 G. McHale, C. V. Brown and N. Sampara, Nat. Commun., 2013, 4, 1605.

33 D. Tian, L. He, N. Zhang, X. Zheng, Y. Dou, X. Zhang, Z. Guo and L. Jiang, Adv. Funct. Mater., 2016, 26, 7986-7992.

34 F. Mugele and J. C. Baret, J. Phys.: Condens. Matter, 2005, 17, R705-R774.

35 A. W. Adamson and A. P. Gast, Physical chemistry of surfaces, Interscience, New York, 1967.

36 M. Vallet, B. Berge and L. Vovelle, Polymer, 1996, 37, 2465-2470.

37 T. B. Jones, K. L. Wang and D. J. Yao, Langmuir, 2004, 20, 2813-2818.

38 T. B. Jones, J. Micromech. Microeng., 2005, 15, 1184-1187.

39 F. Mugele and J. Buehrle, J. Phys.: Condens. Matter, 2007, 19, 375112.

40 A. I. Drygiannakis, A. G. Papathanasiou and A. G. Boudouvis, Langmuir, 2009, 25, 147-152.

41 A. G. Papathanasiou and A. G. Boudouvis, Appl. Phys. Lett., 2005, 86, 164102.

42 J. T. Kedzierski, R. Batra, S. Berry, I. Guha and B. Abedian, J. Appl. Phys., 2013, 114, 024901.

43 M. Vallet, M. Vallade and B. Berge, Eur. Phys. J. B, 1999, 11, 583-591.

44 J. Liu, M. Wang, S. Chen and M. O. Robbins, Phys. Rev. Lett., 2012, 108, 216101.

45 T. Yamamoto, M. Doi and D. Andelman, EPL, 2015, 112, 56001.

46 S. Chevalliot, S. Kuiper and J. Heikenfeld, J. Adhes. Sci. Technol., 2012, 26, 1909-1930.

47 M. Liu, S. Wang and L. Jiang, Nat. Rev. Mater., 2017, 2, 17036.

48 N. Verplanck, E. Galopin, J.-C. Camart, V. Thomy, Y. Coffinier and R. Boukherroub, Nano Lett., 2007, 7, 813-817.

49 Z. Wang, Y. Ou, T.-M. Lu and N. Koratkar, J. Phys. Chem. B, 2007, 111, 4296-4299. 
50 D. L. Herbertson, C. R. Evans, N. J. Shirtcliffe, G. McHale and M. I. Newton, Sens. Actuators, A, 2006, 130, 189-193.

51 C. P. Tan and H. G. Craighead, Materials, 2010, 3, 1803-1832.

52 L. Zhu, J. Xu, Y. Xiu, Y. Sun, D. W. Hess and C.-P. Wong, J. Phys. Chem. B, 2006, 110, 15945-15950.

53 T. N. Krupenkin, J. A. Taylor, E. N. Wang, P. Kolodner, A. Marc Hodes and T. R. Salamon, Langmuir, 2007, 23, 9128-9133.

54 J. L. Campbell, M. Breedon, K. Latham and K. KalantarZadeh, Langmuir, 2008, 24, 5091-5098.

55 F. Lapierre, P. Brunet, Y. Coffinier, V. Thomy, R. Blossey and R. Boukherroub, Faraday Discuss., 2010, 146, 125-139.

56 J.-T. Feng, F.-C. Wang and Y.-P. Zhao, Biomicrofluidics, 2009, 3, 022406.

57 K. S. Teh, Y. Takahashi, Z. Yao and Y.-W. Lu, Sens. Actuators, A, 2009, 155, 113-119.

58 F. Lapierre, V. Thomy, Y. Coffinier, R. Blossey and R. Boukherroub, Langmuir, 2009, 25, 6551-6558.

59 L. Choongyeop and K. Chang-Jin, Phys. Rev. Lett., 2011, 106, 014502.

60 J. B. Boreyko and C.-H. Chen, Phys. Rev. Lett., 2009, 103, 174502.

61 A. G. Papathanasiou, Curr. Opin. Colloid Interface Sci., 2018, 36, 70-77.

62 T. Verho, J. T. Korhonen, L. Sainiemi, V. Jokinen, C. Bower, K. Franze, S. Franssila, P. Andrew, O. Ikkala and R. H. Ras, Proc. Natl. Acad. Sci. U. S. A., 2012, 109, 10210-10213.

63 P. Y. Chiou, S.-Y. Park and M. C. Wu, Appl. Phys. Lett., 2008, 93, 221110.

64 H.-S. Chuang, A. Kumar and S. T. Wereley, Appl. Phys. Lett., 2008, 93, 064104.

65 D. Tian, J. Zhai, Y. Song and L. Jiang, Adv. Funct. Mater., 2011, 21, 4519-4526.

66 Z. Guo, X. Zhang, X. Zheng, Z. Liu, J. Cai, D. Tian, W. Li, J. Zhai, Y. Song and L. Jiang, J. Mater. Chem. A, 2014, 2, 2498-2503.

67 Z. Guo, X. Zheng, D. Tian, Y. Song, J. Zhai, X. Zhang, W. Li, X. Wang, S. Dou and L. Jiang, Nanoscale, 2014, 6, 12822-12827.

68 D. Tian, Y. Song and L. Jiang, Chem. Soc. Rev., 2013, 42, 5184-5209.

69 P. Ferraro, S. Grilli, L. Miccio and V. Vespini, Appl. Phys. Lett., 2008, 92, 213107.

70 W. C. Nelson and C.-J. C. Kim, J. Adhes. Sci. Technol., 2012, 26, 1747-1771.

71 M. G. Pollack, A. D. Shenderov and R. B. Fair, Lab Chip, 2002, 2, 96-101.

72 S. K. Cho, H. J. Moon and C. J. Kim, J. Microelectromech. Syst., 2003, 12, 70-80.

73 H. Song, D. L. Chen and R. F. Ismagilov, Angew. Chem., Int. Ed., 2006, 45, 7336-7356.

74 D. Chatterjee, B. Hetayothin, A. R. Wheeler, D. J. King and R. L. Garrell, Lab Chip, 2006, 6, 199-206.

75 R. Baviere, J. Boutet and Y. Fouillet, Microfluid. Nanofluid., 2008, 4, 287-294.
76 J. Hong, Y. K. Kim, K. H. Kang, J. Kim and S. J. Lee, Sens. Actuators, B, 2014, 190, 48-54.

77 J. Restolho, J. L. Mata and B. Saramago, J. Phys. Chem. C, 2009, 113, 9321-9327.

78 Y. S. Nanayakkara, H. Moon, T. Payagala, A. B. Wijeratne, J. A. Crank, P. S. Sharma and D. W. Armstrong, Anal. Chem., 2008, 80, 7690-7698.

79 H. Li, M. Paneru, R. Sedev and J. Ralston, Langmuir, 2013, 29, 2631-2639.

80 C. W. Monroe, L. I. Daikhin, M. Urbakh and A. A. Kornyshev, J. Phys.: Condens. Matter, 2006, 18, 2837-2869.

81 C. W. Monroe, M. Urbakh and A. A. Kornyshev, J. Phys.: Condens. Matter, 2007, 19, 375113.

82 H. H. J. Girault and D. J. Schiffrin, J. Electroanal. Chem., 1985, 195, 213-227.

83 C. Quilliet and B. Berge, Europhys. Lett., 2002, 60, 99-105. 84 A. Staicu and F. Mugele, Phys. Rev. Lett., 2006, 97, 167801.

85 H. J. J. Verheijen and M. W. J. Prins, Langmuir, 1999, 15, 6616-6620.

86 R. A. Hayes and B. J. Feenstra, Nature, 2003, 425, 383-385.

87 M. Hagedon, S. Yang, A. Russell and J. Heikenfeld, Nat. Commun., 2012, 3, 1173.

88 J. Heikenfeld, K. Zhou, E. Kreit, B. Raj, S. Yang, B. Sun, A. Milarcik, L. Clapp and R. Schwartz, Nat. Photonics, 2009, 3, 292-296.

89 D. Brassard, L. Malic, F. Normandin, M. Tabrizian and T. Veres, Lab Chip, 2008, 8, 1342-1349.

90 C. Hao, Y. Liu, X. Chen, Y. He, Q. Li, K. Y. Li and Z. Wang, Sci. Rep., 2014, 4, 6846.

91 X. He, W. Qiang, C. Du, Q. Shao, X. Zhang and Y. Deng, J. Mater. Chem. A, 2017, 5, 19159-19167.

92 J. Cao, Q. An, Z. Liu, M. Jin, Z. Yan, W. Lin, L. Chen, P. Li, X. Wang, G. Zhou and L. Shui, Sens. Actuators, B, 2019, 291, 470-477.

93 Y. Yan, Z. Guo, X. Zhang, L. He, Y. Li, K. Liu, J. Cai, D. Tian and L. Jiang, Adv. Funct. Mater., 2018, 28, 1800775.

94 J. Hong and S. J. Lee, Lab Chip, 2015, 15, 900-907.

95 S. K. Chung and S. K. Cho, J. Micromech. Microeng., 2008, 18, 125024.

96 S. K. Chung, Y. Zhao and S. K. Cho, J. Micromech. Microeng., 2008, 18, 095009.

97 S. Wang, H. H. Chen and C. L. Chen, Appl. Phys. Lett., 2016, 108, 181601.

98 H. J. Cho, J. P. Mizerak and E. N. Wang, Nat. Commun., 2015, 6, 8599.

99 K. H. Kang, Langmuir, 2002, 18, 10318-10322.

100 E. Colgate and H. Matsumoto, J. Vac. Sci. Technol., A, 1990, 8, 3625-3633.

101 U.-C. Yi and C.-J. Kim, J. Micromech. Microeng., 2006, 16, 2053-2059.

102 J. Kim and M. Kaviany, J. Appl. Phys., 2007, 101, 103520.

103 S. Datta, M. Sharma and A. K. Das, Ind. Eng. Chem. Res., 2014, 53, 6685-6693.

104 S. Datta, A. K. Das and P. K. Das, Langmuir, 2015, 31, 10190-10197. 
105 S. Datta, A. K. Das and P. K. Das, Ind. Eng. Chem. Res., 2016, 55, 3949-3959.

106 S. Datta, A. K. Das and P. K. Das, in Fluid Mechanics and Fluid Power - Contemporary Research, ed. A. K. Saha, D. Das, R. Srivastava, P. K. Panigrahi and K. Muralidhar, 2017, pp. 1305-1313, DOI: 10.1007/978-81-322-2743-4_124.

107 J. Lee and C. J. Kim, J. Microelectromech. Syst., 2000, 9, 171-180.

108 Z. Wan, H. Zeng and A. Feinerman, J. Fluids Eng., 2007, 129, 388-394.

109 M. R. Khan, C. Trlica, J.-H. So, M. Valeri and M. D. Dickey, ACS Appl. Mater. Interfaces, 2014, 6, 22467-22473.

110 S.-Y. Tang, K. Khoshmanesh, V. Sivan, P. Petersen, A. P. O'Mullane, D. Abbott, A. Mitchell and K. Kalantarzadeh, Proc. Natl. Acad. Sci. U. S. A., 2014, 111, 3304-3309.

111 J. Y. Zhu, S.-Y. Tang, K. Khoshmanesh and K. Ghorbani, ACS Appl. Mater. Interfaces, 2016, 8, 2173-2180.

112 J. Wissman, M. D. Dickey and C. Majidi, Adv. Sci., 2017, 4, 1700169.

113 M. Gunji and M. Washizu, J. Phys. D: Appl. Phys., 2005, 38, 2417.

114 J. M. Oh, S. H. Ko and K. H. Kang, Langmuir, 2008, 24, 8379-8386.

115 S. H. Ko, H. Lee and K. H. Kang, Langmuir, 2008, 24, 1094-1101.

116 P. Garcia-Sanchez, A. Ramos and F. Mugele, Phys. Rev. E: Stat., Nonlinear, Soft Matter Phys., 2010, 81, 015303.

117 A. Klingner, S. Herminghaus and F. Mugele, Appl. Phys. Lett., 2003, 82, 4187-4189.

118 J. C. Baret and F. Mugele, Phys. Rev. Lett., 2006, 96, 016106.

119 F. Mugele, J. C. Baret and D. Steinhauser, Appl. Phys. Lett., 2006, 88, 204106.

120 A. Vasudev and J. Zhe, in 2008 17th Biennial University/ Government/Industry Micro-Nano Symposium, Proceedings, IEEE, 2008, pp. 6-10, DOI: 10.1109/ugim.2008.9.

121 A. Vasudev and J. Zhe, in 2008 51st Midwest Symposium on Circuits and Systems, IEEE, 2008, vol. 1 and 2, pp. 49-52, DOI: $10.1109 /$ mwscas.2008.4616733.

122 A. Vasudev and J. Zhea, Appl. Phys. Lett., 2008, 93, 103503.

123 A. Al Amin, A. Jagtiani, A. Vasudev, J. Hu and J. Zhe, J. Micromech. Microeng., 2011, 21, 125025.
124 X.-D. Zhao, H.-M. Fan, J. Luo, J. Ding, X.-Y. Liu, B.-S. Zou and Y.-P. Feng, Adv. Funct. Mater., 2011, 21, 184-190.

125 V. A. Lifton, J. A. Taylor, B. Vyas, P. Kolodner, R. Cirelli, N. Basavanhally, A. Papazian, R. Frahm, S. Simon and T. Krupenkin, Appl. Phys. Lett., 2008, 93, 043112.

126 V. A. Lifton and S. Simon, J. Microelectromech. Syst., 2011, 20, 73-82.

127 C. K. W. Koo, F. He and S. R. Nugen, Analyst, 2013, 138, 4998-5004.

128 R. Tabassian, J.-H. Oh, S. Kim, D. Kim, S. Ryu, S.-M. Cho, N. Koratkar and I.-K. Oh, Nat. Commun., 2016, 7, 13345.

129 G. Kwon, A. K. Kota, Y. Li, A. Sohani, J. M. Mabry and A. Tuteja, Adv. Mater., 2012, 24, 3666-3671.

130 X. Lin, F. Lu, Y. Chen, N. Liu, Y. Cao, L. Xu, W. Zhang and L. Feng, Chem. Commun., 2015, 51, 16237-16240.

131 X. Zheng, Z. Guo, D. Tian, X. Zhang and L. Jiang, Adv. Mater. Interfaces, 2016, 3, 1600461.

132 S. J. Lee, S. Lee and K. H. Kang, J. Visualization, 2011, 14, 259-264.

133 S. J. Lee, S. Lee and K. H. Kang, Appl. Phys. Lett., 2012, 100, 081604.

134 D. Baratian, R. Dey, H. Hoek, D. van den Ende and F. Mugele, Phys. Rev. Lett., 2018, 120, 214502.

135 Y. Chen, N. Doshi, B. Goldberg, H. Wang and R. J. Wood, Nat. Commun., 2018, 9, 2495.

136 L. Mahadevan and D. Vella, Am. J. Phys., 2012, 73, 817-825. 137 D. L. Hu and J. W. M. Bush, Nature, 2005, 437, 733-736.

138 D. L. Hu, M. Prakash, B. Chan and J. W. M. Bush, Exp. Fluids, 2007, 43, 769-778.

139 Y. Yu, M. Guo, X. Li and Q. S. Zheng, Langmuir, 2007, 23, 10546-10550.

140 J. Yuan, J. Feng and S. K. Cho, Langmuir, 2015, 31, 8502-8511.

141 J. M. Carballo, Q. Ni, J. Vasquez and N. B. Crane, Colloids Surf., A, 2017, 512, 118-128.

142 R. D. Deegan, O. Bakajin, T. F. Dupont, G. Huber, S. R. Nagel and T. A. J. N. Witten, Nature, 1997, 389, 827.

143 H. B. Eral, D. M. Augustine, M. H. G. Duits and F. Mugele, Soft Matter, 2011, 7, 4954-4958.

144 K. Carpenter and V. Bahadur, Langmuir, 2015, 31, 2243-2248. 145 F. Yang, R. A. Shaw, C. W. Gurganus, S. K. Chong and Y. K. Yap, Appl. Phys. Lett., 2015, 107, 264101. 